In the remaining 10 patients, histology revealed cirrhosis with active steatohepatitis $(n=2)$, chronic viral hepatitis $(n=1)$ and mild steatosis $(n=7)$. One patient with steatohepatitis was known to be drinking at the time of assessment; the remainder were listed for transplant. Subsequently, one patient with steatosis was found to be drinking and removed. To date, six have been transplanted and survived to 1 year, with two returning to alcohol post-transplant.

Conclusion Histological analysis during liver transplant assessment does not determine the decision to list for liver transplantation nor predict the risk of recidivism. As a result, we no longer routinely perform liver biopsy during liver transplant assessment. A significant proportion of patients return to alcohol consumption post liver transplantation.

\section{P82 HAEMOGLOBIN LEVEL PRIOR TO LIVER TRANSPLANTATION: DOES IT PREDICT THE OUTCOME?}

doi:10.1136/gutjn-2011-300857a.82

${ }^{1} \mathrm{~N}$ Kamperidis, ${ }^{1} \mathrm{M}$ A B Al-Freah, ${ }^{1} \mathrm{M}$ A Heneghan, ${ }^{1} \mathrm{~N}$ Heaton, ${ }^{2} \mathrm{~J} \mathrm{R}$ Goodhand, ${ }^{1} \mathrm{~J}$ O'Grady. ${ }^{1}$ King's College Hospital Liver Unit; ${ }^{2}$ Barts and The London SMD, Center for digestive diseases

Introduction Approximately $75 \%$ of patients with chronic liver disease have anaemia secondary to iron deficiency, haemorrhage, haemolysis, reduced erythropoiesis, hypersplenism, drugs or haemodilution. In other specialties (cardiac and renal) it has been proven that pre-transplant anaemia impacts negatively on both patient and graft survival. This has not been studied in liver graft recipients.

Aim To define whether pre-transplant $\mathrm{Hb}$ levels impact on the 5 -year survival and the need for re-transplantation.

Method Retrospective study of all patients who had liver transplantation (LT) at our centre between 1 August 1988 and 1 August 1999. Patients were included if they had a pre-LT haemoglobin $(\mathrm{Hb})$ level recorded and 5-year follow-up available. $\mathrm{Hb}$ is expressed in $\mathrm{g} / \mathrm{dl}$. In order to define a survival threshold, patients were classified according to their $\mathrm{Hb}$ levels $(\mathrm{Hb}<8, \mathrm{Hb}=8-10, \mathrm{Hb}=10-12$, $\mathrm{Hb}>12)$. Statistical analysis was performed using $\chi^{2}$ test, Kaplan-Meier and Student t test.

Results 720 patients were included. Mean age [SEM] was 37 [0.6]. Males were 349/720 (49\%). 198 patients had $\mathrm{Hb}>12 \mathrm{~g} / \mathrm{dl}, 318$ had $\mathrm{Hb}$ between 10 and $12 \mathrm{~g} / \mathrm{dl}, 182 \mathrm{had} \mathrm{Hb} 8-10 \mathrm{~g} / \mathrm{dl}$ and $22 \mathrm{had} \mathrm{Hb}$ $<8 \mathrm{~g} / \mathrm{dl}$. Mean age of each group was 41 [1.1], 37 [1.0], 34 [1.3], 24 [3.2] $(\mathrm{p}<0.01)$ respectively. The 5 -year survival was $60 \%, 63 \%, 50 \%$

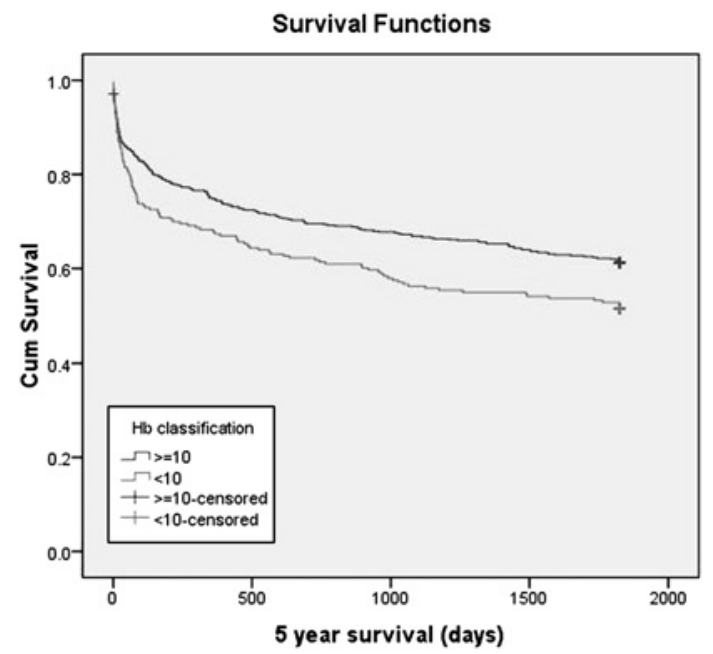

Abstract P82 Figure $1 \quad 5$-year survival post liver transplantation according to $\mathrm{Hb}$ classification $(\mathrm{p}<0.01)$. and $59 \%$ respectively $(p<0.05)$. There was no difference between these $\mathrm{Hb}$ groups in relation to the need for retransplant. Using a $\mathrm{Hb}$ level of $10 \mathrm{~g} / \mathrm{dl}$ as a cut-off value, a Kaplan-Meier survival analysis (see Abstract P82 figure 1) showed that survival was significantly improved in patients with a pre-LT level of $\geq 10 \mathrm{~g} / \mathrm{dl}(\mathrm{p}<0.01)$. Simultaneous multi-variate binary logistic regression [OR, (CI) $\mathrm{p}$ value] showed that age [0.98, (0.97 to 0.99), $<0.01], \mathrm{Hb} \geq 10$ [1.6, (1.2 to 2.3), <0.01] and a diagnosis of PBC [2.5, (1.3 to 4.6), <0.01] could predict the 5 -year survival post-LT.

Conclusion This study demonstrates a significantly worse 5-year survival in patients with pre-LT $\mathrm{Hb}<10 \mathrm{~g} / \mathrm{dl}$. Prospective randomised trials to study the impact of correction of pre-LT $\mathrm{Hb}<10 \mathrm{~g} / \mathrm{dl}$ on the long-term survival is required to define a treatment strategy.

\section{P83 COMBINED LIVER AND KIDNEY TRANSPLANTATION: A SINGLE CENTRE EXPERIENCE}

doi:10.1136/gutjnl-2011-300857a.83

M Smith, K Rye, T Haldane, B Gunson, S Bramhall, D Mutimer. Liver Unit, Queen Elizabeth Hospital Birmingham

Introduction Combined liver kidney transplantation (CLKT) is an accepted approach to management of patients with dual organ pathology, but may be associated with significant additional postoperative morbidity and mortality in comparison with transplantation of either organ alone.

Aim To analyse the experience of CLKT at a single centre.

Method Retrospective analysis of all CLKTs performed at our centre between May 1994 and August 2010. Data collected included demographics, indications for CLKT, surgical techniques, posttransplant complications and patient/graft survival.

Results Of 2130 liver transplants (LT) performed there were 24 CLKTs-12 male with median age 52 years, Child-Pugh score 7 and MELD 19. Indications for LT were polycystic liver disease 11 (46\%), cirrhosis 7 (29\%), hepatocellular carcinoma 2 (8\%), recurrent PSC/PBC 3 (12\%), oxalosis 1 (4\%). The indications for kidney transplantation (KT) were polycystic kidney disease $10(42 \%)$, calcineurin-inhibitor toxicity $4(17 \%)$, chronic kidney graft failure 4 (17\%), IgA nephropathy $3(13 \%)$, diabetes 1 (4\%), Type II hyperoxaluria 1 (4\%), glomerulonephritis 1 (4\%). Five patients had prior KT; four chronic graft failure, 1 calcineurin-inhibitor toxicity. 12 patients (50\%) were dialysis-dependent pre-transplantation.

During a median follow-up of 1223 days (IOR 550-2264 days) 5 patients died (overall survival $79.2 \%$ ) with a median time from CLKT to death 947 days (range 4-2373 days). Causes of death: primary non-function of the liver (1), cardiac complications (2) and de novo cancer (2). Seven patients (29\%) had at least one episode of histologically proven acute cellular rejection of the liver and $1(4 \%)$ acute renal rejection.

Cumulative 1, 3 and 5-year patient, liver graft and kidney graft survival were $96 \%, 85 \%, 75 \% ; 86 \%, 79 \%, 62 \%$ and $91 \%, 85 \%, 75 \%$ respectively. 3 patients required further liver transplantation (2 hepatic artery thrombosis, 1 primary non-function). 13 patients required haemodialysis post-operatively. At 3-month follow-up, survivors had median creatinine and eGFR of $129 \mu \mathrm{mol} / 1$ and $48 \mathrm{ml} /$ min respectively; 1 patient still required dialysis. At 1 year and 5 years median creatinine and eGFR were $134 \mu \mathrm{mol} / 1$ and $43 \mathrm{ml} /$ $\mathrm{min}, 155 \mu \mathrm{mol} / \mathrm{l}$ and $38 \mathrm{ml} / \mathrm{min}$ respectively. 1 patient resumed haemodialysis 4640 days after CLKT and is on the waiting list for renal re-transplantation.

Conclusion CLKT in this cohort had favourable outcomes with excellent patient and graft survival (both organs). Although the number of patients in our study is relatively few, 5 -year kidney graft survival rates do not appear inferior to published data for patients undergoing renal transplantation alone. 